INPLASY

PROTOCOL

To cite: Xiong et al.

Comparative efficacy and safety of statins for osteoporosis: A systematic review and network metaanalysis. Inplasy protocol 202130045. doi:

10.37766/inplasy2021.3.0045

Received: 14 March 2021

Published: 15 March 2021

Corresponding author:

Mengxin Xiong

Rimo.X@outlook.com

Author Affiliation:

Hubei University of Chinese Medicine

Support: NSFC (NO.82074416;

NO.81904267).

Review Stage at time of this submission: Piloting of the study selection process.

Conflicts of interest:

None declared.

\section{Comparative efficacy and safety of statins for osteoporosis: A systematic review and network meta-analysis}

Xiong, MX1; Xiang, N2; Zhou, GW3; Zhu, W4; Xue, YJ5; Tan, ZK6; Deng, $A^{7}$.

Review question / Objective: We conducted this systematic review and network-meta analysis to evaluate the comparative effectiveness of statins, including the risk of fracture, bone mineral density(BDM), the markers of bone metabolism, and adverse events.

Information sources: We will conduct electronic searches via MEDLINE, EMBASE , Web of Science, Cochrane Central Resister of Controlled Trials (CENTRAL), China National Knowledge Infrastructure (CNKI), Wanfang Database, China Science and Technology Journal Database (VIP), and Chinese Biomedical Literature Database (CBM) from from their inception to May 31, 2021. We will search ongoing trials via the World Health Organization International Clinical Trials Registry Platform (http://apps.who.int/trialsearch/) and ClinicalTrials.gov (https://ClinicalTrials.gov/) on May 31, 2021, without date limits and language restrictions. We will scan the bibliographies of all retrieved trials and those of existing systematic reviews and meta-analyses relevant to randomized controlled trials for the current review question.

INPLASY registration number: This protocol was registered with the International Platform of Registered Systematic Review and Meta-Analysis Protocols (INPLASY) on 15 March 2021 and was last updated on 15 March 2021 (registration number INPLASY202130045).

\section{INTRODUCTION}

Review question / Objective: We conducted this systematic review and network-meta analysis to evaluate the comparative effectiveness of statins, including the risk of fracture, bone mineral density(BDM), the markers of bone metabolism, and adverse events.

Condition being studied: Osteoporosis is prevalent skeletal disease characterized by low bone mass, microarchitectural changes in bones, and skeletal fragility. These changes result in decreased bone 
strength and an increased propensity for fractures. Osteoporosis usually decreases life quality because of back pain and disability, especially in older people. The most preventative and curative drugs for osteoporosis work through the antiresorptive or the anabolic mechanism. Those drugs have certain clinical use limitations due to their medication method(such as teriparatide is given mainly by subcutaneous injection ), medication holidays, or cost issues, though they have promising efficacy. Thus, it is essential to discover new drugs. The 3-hydroxy-3methylglutarylcoenzyme $A$ reductase inhibitors (statins) have become a mainstay in preventing and treating cardiovascular disease (CVD), and they appear to be potentially promising drugs for osteoporosis. Previous studies have shown the efficacy of statins in osteoporosis, though controversial. Studies have shown that the diagnosis of osteoporosis in statintreated patients is dose-dependent, which statins are more effective and safety at this time is unclear.

\section{METHODS}

Participant or population: Inclusion Criteria:We will include adults with a medical diagnosis of osteoporosis, and statins are the main treatment drugs. Exclusion Criteria: Studies that do not list basic population demographics (i.e. Age and Gender). Studies involving paediatric and adolescent populations. Studies involving healthy, asymptomatic patients or populations. Patients with osteoporosis caused by other diseases or drugs. Studies with their follow-up period< 2 months.

Intervention: We will include studies setting statins or "HMG-CoA reductase inhibitors including lovastatin, pravastatin, simvastatin, fluvastatin, atorvastatin, rosuvastatin and pitavastatin as interventions.

Comparator: To evaluate statins' effectiveness, no intervention or placebo or calcium and/or vitamin $D$ as adjuvant therapy will be set as a control.
Study designs to be included: Randomized trials, regardless of published or unpublished, will be included without language and data restriction.

Eligibility criteria: Studies were eligible for this review if they met the following criteria: they (i) were randomized controlled trials; (ii) enrolled adult patients diagnosed with osteoporosis; (iii) compared one or more of the interventions of interest to each other or to placebo; (iv) reported at least one outcome of interest (fractures, bone mineral density, bone turnover marker, adverse events ) as a primary or secondary outcome or as an adverse event; and (vi) participants in treatment group were treated with statins.

Information sources: We will conduct electronic searches via MEDLINE, EMBASE , Web of Science, Cochrane Central Resister of Controlled Trials (CENTRAL), China National Knowledge Infrastructure (CNKI), Wanfang Database, China Science and Technology Journal Database (VIP), and Chinese Biomedical Literature Database (CBM) from from their inception to May 31, 2021. We will search ongoing trials via the World Health Organization International Clinical Trials Registry Platform (http://apps.who.int/trialsearch/) and ClinicalTrials.gov (https:// ClinicalTrials.gov/) on May 31, 2021, without date limits and language restrictions. We will scan the bibliographies of all retrieved trials and those of existing systematic reviews and meta-analyses relevant to randomized controlled trials for the current review question.

Main outcome(s): The primary outcomes are the incidence of fractures overall fracture and the improvement of BDM (percentage change and absolute change [in $\mathrm{g} / \mathrm{cm} 2$ ]) at the lumbar spine, the femoral neck or the hip. Measures of effect: Record and compare the risk of fractures. BDM were measured by dualenergy X-ray absorptiometry (DEXA).

Additional outcome(s): Secondary outcomes included adverse effects such as 
gastrointestinal or liver side effects of therapy and changes in bone turnover markers. Measures of effect :Record and compare the adverse effects. Bone turnover markers include $\mathrm{N}$-terminal propeptide of type I procollagen (PINP); Cterminal telopeptide of type I collagen (CTX); crosslinked N-telopeptide of type I collagen (NTX); alkaline phosphatase(AP).

Data management: We will conduct a systematic literature search using the searching strategies described above to obtain the titles and abstracts of candidate studies of this review. Four authors (X-MX, Z-W, Z-GW, X-YJ) will be divided into two teams and each team will review the half of candidate articles. The titles and abstracts will be examined independently by two authors within the same team according to the inclusion/exclusion criteria and the articles which will be considered to meet the criteria will be reassessed by the same process after obtaining their full articles. In the case of discordance of author's opinion within one team, one author from the other team will assess the inclusion of articles with the discordance. To assess the inclusion of articles published only as abstracts, we will contact the authors of these studies to request their details. To extract the required data for data synthesis from the selected articles, we will use a structured Excel form designed by the collaboration of four authors as follows: setting, number of participants with baseline characteristics, details of interventions (kinds of medication, dose, duration), primary and secondary outcomes with follow-up periods and number of withdrawals in each group with reasons. If data in the published articles are insufficient, we will contact the authors of selected articles to necessary data.

Quality assessment / Risk of bias analysis: The following items referenced by Cochrane risk of bias assessment tool will be assessed independently by two teams. Each domain will be categorized as high risk, low risk and unclear. In the case of discordance among the authors within one team, one author from the other team will assess the categorization, which will be the same process as the selection of articles. 1. Was there adequate sequence generation (selection bias)? 2. Was allocation adequately concealed (selection bias)? 3. Was knowledge of the allocated interventions adequately prevented during the study? 4. Participants and personnel (performance bias) 5. Outcome assessors (detection bias) 6. Were incomplete outcome data adequately addressed (attrition bias)? 7. Are reports of the study free of suggestion of selective outcome reporting (reporting bias)? 8. Was the study apparently free of other problems that could put it at a risk of bias? The quality of evidence for each outcome will be rated from high to very low according to the GRADE system providing the reasons to downgrade the quality as below: 1. Risk of bias 2 . Inconsistency 3 . Indirectness 4. Imprecision 5. Publication bias.

Strategy of data synthesis: First, we will do a traditional pairwise meta-analysis, which is used for consistency check and an evaluation of heterogeneity, for all available direct evidence comparing two treatments using Stata, V.13.0 (Stata Corp, College Station, Texas, USA). The 12 statistic will be applied to quantify the extent of betweentrial heterogeneity, with $I 2>50 \%$ indicating considerable heterogeneity. The randomeffects model will be used as the main model. Furthermore, the results of the random-effects model will be compared with that of the fixed-effects model to test the stability of the results. OR with $95 \% \mathrm{CI}$ will be calculated for a dichotomous variable. Mean difference (MD) with $95 \% \mathrm{CI}$ will be estimated for a continuous outcome. Network meta-analysis will be conducted using a Bayesian Markov chain Monte Carlo (MCMC) framework and fitted in $R$ V.3.2.4 software (https://cran. $r$ project.org/src/base/R-3/) via the gemtc V.0.81 package. A Gaussian model will be used for the continuous variables, and a Bernoulli model will be used for the dichotomous variables. The posterior distribution of the parameter which is used for inference will be summarised by its median (OR or MD) and $95 \%$ credible interval (Crl). Three chains with different 
initial values will be run simultaneously. For the analyses, inference will be based on 150000 iterations of MCMC after a 50000 iteration burn-in period.23 To assess convergence, trace plots and BrooksGelman-Rubin diagnostic plots will be used.

Subgroup analysis: If sufficient trials are included, subgroup analyses will be conducted to assess the differences in the effect estimates. We plan to conduct those analyses based on the following subgroups: 1. Different ethnicities; Asian or others 2. Different pathological subtypes: postmenopausal osteoporosis or senile osteoporosis.

Sensitivity analysis: We will perform sensitivity analyses as follows: 1 . Repeating the analysis restricting study with low risk of selection bias (i.e. adequate random sequence generation and random allocation). 2. Exclusion of studies using imputed statistics. 3. Missing participants: Best-best scenario where all missing participants in the two groups remain unchanged $A$ best-worst scenario where all missing participants in the intervention group remain unchanged and all missing participants in the control group have outcomes The worst-best scenario where all missing participants in the intervention group have outcomes and all missing participants in the control group remain unchanged.

Language: None restriction.

Country(ies) involved: China.

Keywords: Osteoporosis; Bone turnover marker; Bone mineral density; Fracture; HMG-CoA reductase inhibitors; Statins.

Contributions of each author:

Author 1 - Mengxin Xiong - The author drafted the manuscript and involved in the data extraction.

Email: Rimo.X@outlook.com

Author 2 - Nan Xiang - The author is responsible for all inspections and proofreading of the review among with Author 7.
Email: xiangnan61@sina.com

Author 3 - Guangwen Zhou - The author participated in the data extraction.

Email: 60905325@qq.com

Author 4 - Wei Zhu - The author participated in the data extraction.

Email: weizhu@whu.edu.cn

Author 5 - Yaojun Xue - The author participated in the data extraction.

Email: 410061687@qq.com

Author 6 - Zhangkui Tan - The author searched literatures.

Email: 446090035@qq.com

Author 7 - Ali Deng - The author is responsible for all inspections and proofreading of the review among with Author 2.

Email: 1556635835@qq.com 